\title{
Fas-associating death domain protein overexpression induces apoptosis in lung cancer cells
}

Peter K. M. Kim, MDa

Sang-Youel Park, PhD

Patrick P. Koty, PhD

Yun Hua, $\mathrm{BA}^{\mathrm{a}}$

James D. Luketich, $M D^{\mathrm{C}}$

Timothy R. Billiar, $\mathrm{MD}^{\mathrm{a}}$

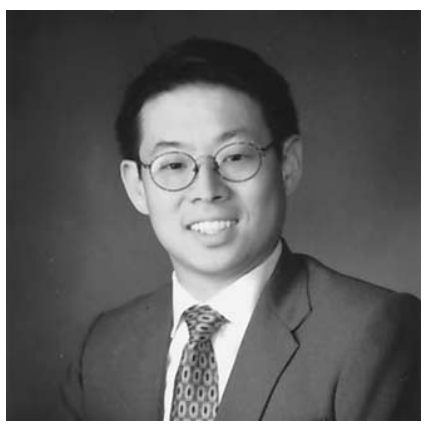

Dr Kim

Objectives: Non-small cell lung cancers commonly develop resistance to radiation and chemotherapy, and they often present at stages beyond surgical resectability. Because current treatment modalities are inadequate, novel therapies are necessary to reduce the effects of the increasing incidence in pulmonary neoplasms. Fasassociating death domain protein is a central mediator of death receptor-initiated apoptosis that directly activates the caspase- 8 protease. We hypothesized that overexpression of Fas-associating death domain protein would effectively eradicate lung cancer cells by induction of apoptosis.

Methods: Cultured A549 alveolar carcinoma and NCI-H226 squamous carcinoma cells were exposed to increasing multiplicities of infection of a replication-deficient, adenoviral vector that expresses the wild-type murine Fas-associating death domain protein gene or control virus for 4 hours. Twenty-four hours later, cells were assessed for viability by crystal violet staining and caspase activation by microscopic analysis. Protein lysates were examined by Western blotting for expression of Fas-associating death domain protein and activated caspase-8.

Results: Adenoviral infection with the wild-type murine Fas-associating death domain protein gene in A549 cells resulted in a dose-dependent expression of Fas-associating death domain protein and the appearance of cleaved, activated caspase-8. Increasing multiplicities of infection of the wild-type murine Fas-associating death domain protein gene, but not control adenovirus, was associated with increased cell death in A549 and NCI-H226 cells. The wild-type murine Fasassociating death domain protein gene infection of A549 cells at multiplicities of infection of 50 induced at least 10-fold increase in Fas-associating death domain protein levels and decreased viability by $>50 \%(\mathrm{n}=3 ; P<.001)$.

Conclusion: Overexpression of Fas-associating death domain protein induced dosedependent cell death in A549 and NCI-H226 lung epithelial cancer cells. Expression of Fas-associating death domain protein results in activation of caspases, a hallmark of apoptosis. Delivery of the wild-type murine Fas-associating death domain protein gene to drug- and radiation-resistant lung cancer may be a novel method for therapy of non-small cell lung cancer.

University of Pittsburgh, Department of Surgery Laboratories, NW607 MUH, 3459 Fifth Ave, Pittsburgh, PA 15213 (E-mail: kimp@msx.upmc.edu).

J Thorac Cardiovasc Surg 2003;125: $1336-42$

Copyright (C) 2003 by The American Association for Thoracic Surgery

$0022-5223 / 2003 \$ 30.00+0$

doi:10.1016/S0022-5223(02)73227-3

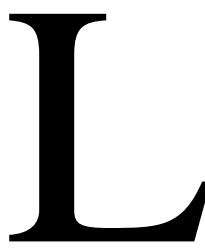

ung cancer is the primary cause of malignancy-associated death in the United States, with 170,000 new cases and 157,000 deaths estimated in 2001. ${ }^{1}$ Neither screening for early disease nor advances in conventional cancer therapies have altered the 14\% 5-year survival rate of patients with non-small cell lung cancer. Therefore, further investigations are needed to identify new strategies for treatment. 
Cellular death by the process of apoptosis is evident morphologically by plasma membrane blebbing, cytoplasmic condensation, and fragmentation of cells and nuclei. ${ }^{2}$ Apoptosis can be induced with radiation, chemotherapeutic agents, or oligomerization of death receptors by their respective death ligands. ${ }^{3,4}$ Fas-associating death domain protein/mediator of receptor-induced toxicity (FADD/MORT1) is a $29 \mathrm{kD}$ cytoplasmic adaptor molecule that mediates signal transduction pathways of apoptosis. ${ }^{5,6}$ When cells are treated with death ligands that bind and activate death receptors, FADD is recruited to the plasma membrane and interacts with the intracellular death domain of death receptors like Fas. ${ }^{7,8}$ FADD then oligomerizes the procaspase- 8 zymogen and induces activation of caspase- 8 by autocleavage. ${ }^{9,10}$ Activated caspase- 8 can then cleave downstream molecules that transmit and amplify the programmed cell death signal. ${ }^{11}$

Because of the central role of FADD in activation of apoptosis, we hypothesized that intracellular overexpression of FADD would bypass death ligands and receptors to induce caspase- 8 activation and apoptosis. To prove this hypothesis, an adenoviral vector that generates FADD protein was constructed and used to induce FADD overexpression in A549 and NCI-H226 lung carcinoma cells. The cells were then assessed for apoptotic structure, cell viability, and activation of caspases.

\section{Materials and Methods \\ Cells and Reagents}

A549 (alveolar cell carcinoma) and NCI-H226 (squamous cell carcinoma) cells were obtained from the American Type Culture Collection (Manassas, Va). A549 cells were maintained in media containing F-12K Nutrient Mixture (Life Technologies, Carlsbad, Calif), supplemented with penicillin $(100 \mathrm{U} / \mathrm{mL})$, streptomycin (100 mg/mL), and $10 \%$ fetal bovine serum (v/v). NCI-H226 cells were grown in RPMI-1640 media with $10 \%$ fetal bovine serum $(\mathrm{v} / \mathrm{v})$, penicillin $(100 \mathrm{U} / \mathrm{mL})$, streptomycin $(100 \mathrm{mg} / \mathrm{mL})$, and L-glutamine (1\%). Antibodies used for this study were purchased from Sigma Chemical Co (for $\alpha$-actin; St Louis, Mo), Stressgen (for caspase-8; Victoria, BC, Canada), and Calbiochem (for FADD; San Diego, Calif). BCA protein and Supersignal chemiluminescence detection reagents were from Pierce (Rockford, Ill). Unless otherwise indicated, all other reagents were purchased from Sigma Chemical Co.

\section{Viral Vectors and Infection}

Ad-FADD was constructed through Cre-lox recombination with reagents provided by Dr S. Hardy (Somatix, Alameda, Calif). An XhoI/Xhol 720 bp murine FADD cDNA coding region (isolated from PCImMort1 FL; generously provided by Dr Astar Winoto) was inserted into the shuttle vector pAdlox to create the Ad-FADD expression vector. Ad-EGFP (enhanced green fluorescent protein) was constructed and obtained as described previously ${ }^{12}$, and AdControl (empty vector) was a gift from S. Hardy.

Cells were plated at $2 \times 10^{5}$ cells per well in 6 -well dishes for Western blotting and $5 \times 10^{4}$ cells per well in 12 -well plates for cell viability. For FADD gene transduction of A549 cells, Ad-FADD was added to cell cultures at increasing multiplicities of infection (MOI = number of PFU per cell) in serum-free Opti-MEM (Gibco BRL, Rockville, Md). Cells were incubated for 4 hours at $37^{\circ} \mathrm{C}$ and $5 \% \mathrm{CO}_{2}$. Cells were washed twice with phosphate-buffered saline solution (PBS) and fresh media containing $10 \%$ serum was added. After 24 hours, cell structure was photographed under phase-contrast light microscopy.

\section{Immunoblotting Analysis}

Cells were washed with cold PBS and removed from plates with $0.25 \%$ trypsin. Cells were resuspended in 5-fold volume of lysis buffer containing $50 \mathrm{mmol} / \mathrm{L}$ Tris $(\mathrm{pH} 7.5), 150 \mathrm{mmol} / \mathrm{L} \mathrm{NaCl}, 1$ $\mathrm{mmol} / \mathrm{L}$ ethylenediamine tetraacetic acid, $1 \%$ Triton $\mathrm{X}-100,0.5$ $\mathrm{mmol} / \mathrm{L}$ phenylmethylsulfonyl fluoride, $5 \mu \mathrm{g} / \mathrm{mL}$ aprotinin, 5 $\mu \mathrm{g} / \mathrm{mL}$ pepstatin, and $10 \mu \mathrm{g} / \mathrm{mL}$ leupeptin. Lysates were incubated at $4^{\circ} \mathrm{C}$ for 30 minutes, and cell debris was removed by centrifugation at $13,000 \times g$ at $4^{\circ} \mathrm{C}$ for 15 minutes. The supernatant was used for Western blotting analysis. Protein concentration was determined with the BCA assay (Pierce) with bovine serum albumin as standard.

Forty micrograms of protein were separated on $13 \%$ SDSPAGE and transferred onto a nitrocellulose membrane. Loading of equal protein amounts was assessed by staining of membranes with $0.1 \%$ Ponceau S (Sigma Chemical Co) in 5\% acetic acid. Nonspecific binding was blocked with PBS-T (14 mmol/L sodium phosphate, monobasic, monohydrate; $88 \mathrm{mmol} / \mathrm{L}$ dibasic sodium phosphate, anhydrous; $100 \mathrm{mmol} / \mathrm{L} \mathrm{NaCl}$; and $0.1 \%$ Tween 20 ) containing $5 \%$ nonfat milk for 1 hour incubation with agitation at room temperature. Membranes were incubated with anti-FADD (1:4000), anti-caspase-8 (1:1000), or anti- $\alpha$-actin (1:1000) antibodies for 1 hour and washed three times with PBS-T. The horseradish peroxidase-conjugated goat antirabbit secondary antibody (Amersham Pharmacia Biotech, Piscataway, NJ) was incubated with membranes at 1:10,000 for 1 hour at room temperature. After five washes with PBS-T, the protein bands were visualized with Supersignal (Pierce) according to the manufacturer's instructions. The bands were visualized on Kodak film (Eastman Kodak, Rochester, NY) exposed to the membrane to detect chemiluminescence signals. Intensity of bands was assessed by densitometry with UN-SCAN-IT (Silk Scientific, Orem, Utah).

\section{Cell Viability Assays}

Cell viability was determined by the crystal violet method as described previously. ${ }^{13}$ Briefly, adherent cells were stained with $0.5 \%$ crystal violet in $30 \%$ ethanol and $3 \%$ formaldehyde for 10 minutes at room temperature. Plates were washed six times with tap water. After drying, cells were lysed with $1 \%$ sodium dodecyl sulfate solution, and dye uptake was measured at $550 \mathrm{nmol} / \mathrm{L}$ with a 96-well microplate reader. Cell viability was calculated from relative dye intensity of the mean for duplicate samples and presented as percentages relative to untreated samples.

\section{Caspase Activity Assay}

Detection of activated caspases in NCI-H226 cells was performed with the CaspaTag Pan-Caspase activity kit (Intergen Co, Purchase, NY), following the manufacturer's protocol. Briefly, 


\title{
Ad-FADD (MOI) $0 \quad 5102550$ \\ FADD - \\ Actin -
}

\begin{abstract}
Figure 1. Dose-dependent FADD protein expression in lung cancer cells infected with Ad-FADD. A549 cells $(2 \times$ $10^{5}$ ) were plated in 6 -well dishes and infected with increasing multiplicities of infection (MOI: $0,5,10,25$, and 50 ) of Ad-FADD. Cells were harvested 24 hours later and protein lysates were probed by Western immunoblotting with anti-FADD antibody. Membranes were stripped and reprobed with anti-actin antibody to demonstrate equal loading of lanes.
\end{abstract}

caspase activation was detected using a fluorometric change upon cleavage of a carboxyfluorescein-labeled fluoromethyl ketone-peptide inhibitor (VAD). Nuclei were stained with Hoechst 33342. Five hundred cells were visualized and counted in duplicate with a triple bypass filter (Chroma 61002).

\section{Statistical Analysis}

Data are presented as means \pm standard error of the mean of three separate experiments, except where results of blots are shown in which case a representative experiment is depicted in the figure. Comparisons between values were analyzed by one-way analysis of variance (ANOVA) by use of the Student-Newman-Keuls method with SigmaStat Statistical Software Version 2.03. Differences were considered significant at $P \leq .05$.

\section{Results}

\section{FADD Expression in A549 Lung Cancer Cells}

To estimate adenoviral infectivity of human lung cancer cells, A549 cells were infected with Ad-EGFP (enhanced green fluorescent protein) control virus. Greater than $90 \%$ of A549 cells were fluorescent after infection with Ad-EGFP at MOI 50, demonstrating efficient infectivity of these cells (data not shown).

To assess whether the Ad-FADD vector could induce expression of FADD protein in lung cancer cells, A549 cells were infected with Ad-FADD in increasing concentrations and harvested 24 hours later to assess FADD expression. Western blotting was performed to demonstrate that infection with Ad-FADD resulted in a dose-dependent increase in FADD expression in A549 lung cancer cells (Figure 1). Ad-FADD at MOI of 50 induced greater than a 10-fold increase in FADD expression. Actin levels were assessed to confirm equal loading of protein in each lane.

\section{Ad-FADD Infection and Dose-Dependent Apoptotic Cell Death}

To assess the structure of cells that overexpress FADD, A549 cells infected with Ad-FADD were observed by phase-contrast microscopy. Increasing doses of Ad-FADD resulted in augmented numbers of cells with typical, apoptotic morphology consisting of detached, condensed cells with membrane blebbing (Figure 2). Control cells infected with Ad-EGFP at MOI 50 (Figure 2, B) or no virus (Figure $2, A$ ) displayed a minimal number of cells with apoptotic structure.

To quantitate the cell death induced by Ad-FADD in A549 cells, cell viability was measured for A549 cells after infection with Ad-EGFP, no virus, or increasing concentrations of Ad-FADD. Twenty-four hours later, cells were fixed and stained with crystal violet, lysed, and quantitated by spectrophotometry at $550 \mathrm{~nm}$ (Figure 3). Compared with control, Ad-EGFP infection resulted in an insignificant decrease in cell viability $(93.4 \pm 6.1 \%, \mathrm{n}=3, P=.271)$. However, Ad-FADD infection induced a dose-dependent increase in cell death compared with Ad-EGFP infection. Infection of A549 cells with Ad-FADD at MOI 10 showed significant decrease in cell viability to $75.2 \pm 5.6 \%(\mathrm{n}=3$, $P=0.02$ ). Ad-FADD treatments at MOI 25 and 50 resulted in further decreases in cell viability to $57.1 \pm 5.8 \%$ and 47.7 $\pm 3.9 \%$, respectively $(\mathrm{n}=3, P<.001)$.

\section{Ad-FADD Treatment- and Concentration-Dependent Caspase-8 Cleavage}

Caspase-8, the most proximal initiator protease in the signaling of apoptosis, exists as an inactive $55 \mathrm{kD}$ zymogen called procaspase- 8 in cells that have not been stimulated to undergo apoptosis. ${ }^{11}$ Treatment of cells with FasL results in cleavage of procaspase-8 into multiple active forms, including a $26-\mathrm{kD}$ cleavage band that can be visualized by Western blotting. To demonstrate that Ad-FADD treatment of lung cancer cells would result in caspase-8 activation, A549 cells were exposed to increasing concentrations of AdFADD, Ad-control virus, or no virus. Twenty-four hours later, cells were harvested and the expression levels of cleaved and uncleaved forms of caspase- 8 were assessed by Western blotting (Figure 4). Lysates of A549 cells from control virus infection (lane 1, Ad-control at MOI 50) and 
no virus treatment (lane 2) showed endogenous levels of procaspase-8 with no expression of the cleaved, activated fragment of caspase- 8 . In contrast, increasing concentrations of Ad-FADD resulted in dose-dependent elevations in active caspase-8, with corresponding reductions in expression of the procaspase- 8 zymogen. The cleavage of caspase- 8 appeared to correlate with the increase in expression of FADD protein (Figure 1), apoptotic structure (Figure 2 ), and the decrease in cell viability (Figure 3 ) observed in A549 cells infected with Ad-FADD.

\section{Ad-FADD Infection-Induced Apoptotic Cell Death and Caspase Activation in NCI-H226 Lung Cancer Cells To demonstrate that the response to Ad-FADD was not idiosyncratic to A549 cells, we treated the NCI-H226 lung squamous carcinoma cell line with Ad-FADD. As in A549 cells, NCI-H226 cells infected with Ad-FADD induced cell death with the characteristic structure of apoptosis, includ- ing cytoplasmic condensation and plasma membrane bleb- bing (Figure 5). Ad-FADD treatment reduced NCI-H226 viability from $100 \%$ to $64 \%$ by 24 hours after infection (Figure 5). Control virus Ad-EGFP resulted in $>90 \%$ infectivity at MOI 50 (data not shown), but did not induce significant cell death. Ad-FADD infection of NCI-H226 cells resulted in caspase activation (Figure 5, red) in a significantly higher percentage of cells compared with con- trols (Ad-FADD $18.8 \pm 5.0 \%$ versus mock virus $2.4 \pm$ $0.3 \%$ versus media $1.8 \pm 0.4 \%, \mathrm{n}=3$, ANOVA $P=.002$ ).}

\section{Discussion}

We have constructed a replication-deficient adenovirus that expressed the FADD death gene in human lung cancer cells and demonstrated that overexpression of FADD in A549 cells induced dose-dependent cell death with apoptotic structure. The mechanism of this cell death involved cleavage of caspase- 8 , the most proximal initiator protease in the signal transduction of apoptosis. This study proves our hypothesis that without the addition of death receptor ligands or chemotherapeutic agents, overexpression of FADD alone could induce apoptotic cell death in human lung cancer cells.

Radiation and many chemotherapeutic agents induce apoptosis in susceptible cancer cells. ${ }^{4,14}$ Nevertheless, nonresponders and recurrence of tumors are associated with drugresistant cancer clones. ${ }^{15,16}$ The induction of apoptosis by ionizing radiation and chemotherapeutic drugs involves either death receptor activation or, more commonly, p53dependent release of proapoptotic stimuli from mitochondria that results in activation of caspase-9 (Figure 6). ${ }^{17,18}$ Significantly, these agents have been shown to induce apoptosis in some cancer cells in the absence of FADD. ${ }^{19}$ However, in p53-deficient cells or cells that overexpress Bcl-2, apoptosis could still be induced by Fas ligand. This

\section{Ad-EGFP Ad-FADD} Media MOI $50 \quad$ MOI 5
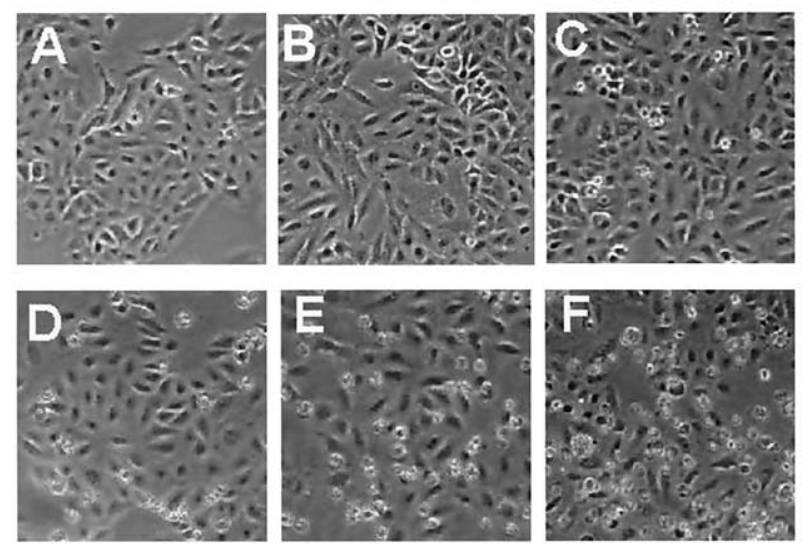

\section{Ad-FADD Ad-FADD Ad-FADD MOI $10 \quad$ MOI $25 \quad$ MOI 50}

Figure 2. Structure of A549 cells treated with Ad-FADD infection. Cells plated in 12 wells were infected with no virus (A), control Ad-EGFP at MOI 50 (B), or Ad-FADD at increasing MOI of 5 (C), 10 (D), 25 (E), and 50 (F). Twenty-four hours after infection, cell structure was photographed under phase-contrast microscopy at original magnification $\times 10$.

finding demonstrated that even when the mitochondrial pathway of cell death was blocked in tumors, the Fas-FADD pathway of cell death is still intact. ${ }^{19}$ Therefore, the therapeutic activation of FADD-dependent cell death pathways may be useful to eradicate cancer cells resistant to radiation or chemotherapy, or in conjunction with these modalities. Unlike A549 cells, which are p53 wild type, NCI-H226 cells contain a missense mutation (R158L) in p53. Because NCI-H226 cells were effectively killed by Ad-FADD (Figure 5), these studies suggest that Ad-FADD can induce apoptosis by a p53-independent mechanism. Because mutations in p53 are the most common mutation in human cancer, overexpression of FADD may be a viable alternative to other therapeutic modalities that require wild-type $\mathrm{p} 53$.

Induction of tumor cell death by overexpression of FADD may have several therapeutic advantages ${ }^{20}$ FADD is an essential signaling molecule for apoptosis induced via both Fas and tumor necrosis factor-related apoptosis-inducing ligand (TRAIL) death receptors. ${ }^{8,21}$ A549 cells are known to have decreased expression of cell surface Fas death receptors and are resistant to apoptosis induced by anti-Fas antibody. ${ }^{22}$ TRAIL is known to be cytotoxic to A549 cells ${ }^{23}$ with some specificity to cancer cells. ${ }^{24}$ However, the cell surface expression of TRAIL death receptors also has been discovered to be downregulated or mutated in 


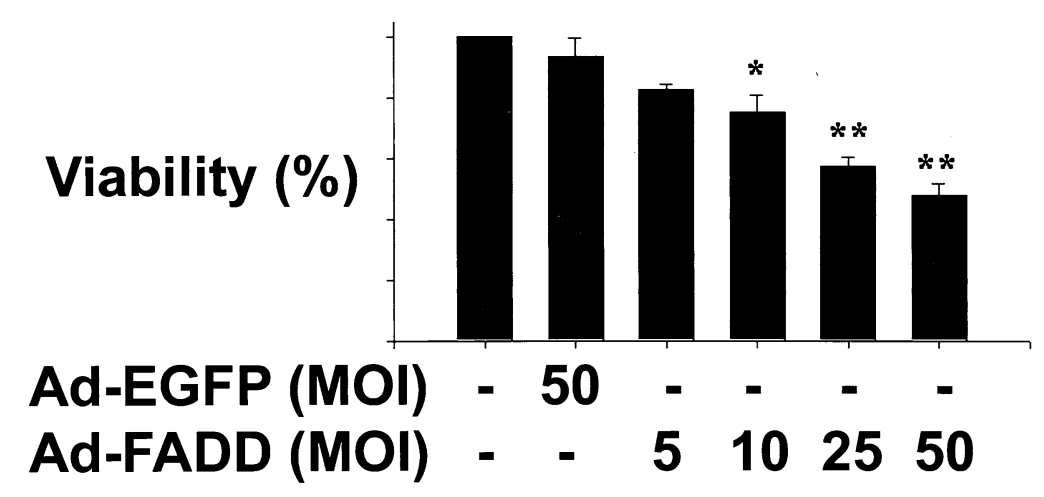

Figure 3. Effect of Ad-FADD infection on A549 cell viability. A549 cells plated in 12 wells were exposed to no virus, Ad-EGFP at MOI 50, or increasing concentrations of Ad-FADD (5, 10, 25, and 50). Twenty-four hours later, cell viability was determined by crystal violet staining. Viability of control cells (no virus) was set at $100 \%$ and viabilities relative to control are presented. Experiments were performed in duplicate for three experiments. Error bars indicate standard error of the means. Significance was assessed with one-way ANOVA comparing the viability after each Ad-FADD infection to the viability after Ad-EGFP infection at MOI 50 ( $n=3,{ }^{*} P=.02,{ }^{* *} P<$ .001).
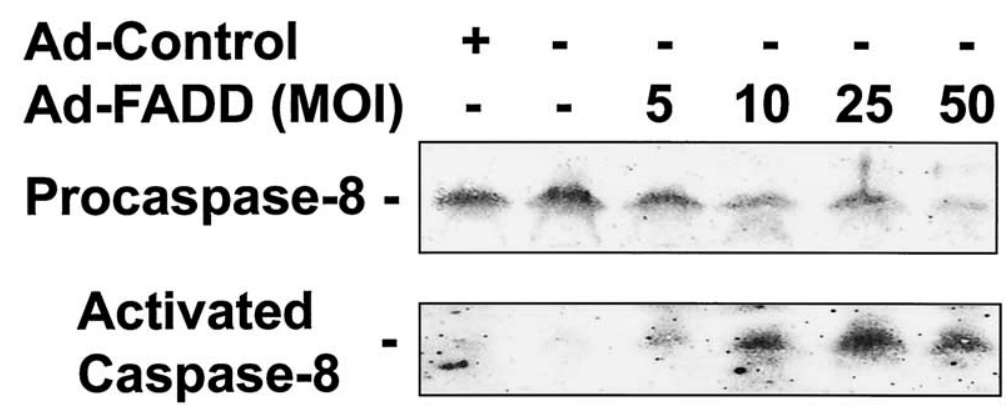

Figure 4. Effect of Ad-FADD infection on procaspase-8 and activated caspase-8 cleavage protein levels. A549 cells were plated in 6-well dishes and infected with increasing multiplicities of infection of Ad-FADD (MOI: 0, 5, 10, 25, and 50), control empty virus (Ad-control) at MOI 50, or no virus. Cells were harvested 24 hours later and lysates were probed by Western immunoblotting with anti-caspase-8 antibody.

some cancer cells, which make them resistant to apoptosis by death ligands. ${ }^{25,26}$ Inactive, decoy death receptors have also been described in lung and colon cancers. ${ }^{27}$ In our experiment, we have demonstrated that overexpression of FADD results in caspase- 8 activation without the addition of FasL or TRAIL, effectively bypassing the need for functional death receptors.

This study was performed to test our hypothesis that overexpression of the FADD protein is a novel method to induce apoptotic cell death in lung tumor cells. The major drawback to these preliminary results includes the lack of confirmation in an animal model of lung cancer. Intratumoral injections or endovascular delivery via a feeding artery would be required to assess the ability of the AdFADD vector to induce tumor cell death in vivo. However, because adenoviral vectors are rapidly cleared by the immune system, intratumoral injections of lung tumors may not support any clinical advantage over other surgical or systemic modalities unless a "bystander effect" can be induced, as seen with gene therapy vectors that express wildtype $\mathrm{p} 53 .{ }^{28}$ Production of a cancer-specific vector ${ }^{29}$ that encodes fadd would be necessary to assess intratumoral FADD expression as a systemic, therapeutic approach that could target lung neoplasms for unresectable or micrometastatic disease. As an alternative to gene therapy, we have demonstrated other modalities to increase endogenous FADD expression in hepatocytes and keratinocytes that do not involve viral vectors. ${ }^{30,31}$ Future research is also required to demonstrate whether lung cancer cell death by overexpression of FADD may be enhanced in combination with chemotherapy and radiation. Modulation of FADD expression may be effective for lung cancer when other modalities have failed, an all too common event experienced in the therapeutic history of lung cancer. 
Media

\section{Viability}

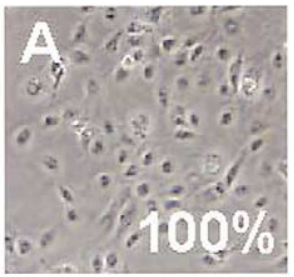

Caspase Activation
Ad-EGFP

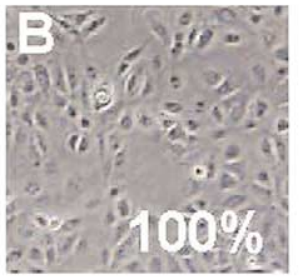

Ad-FADD
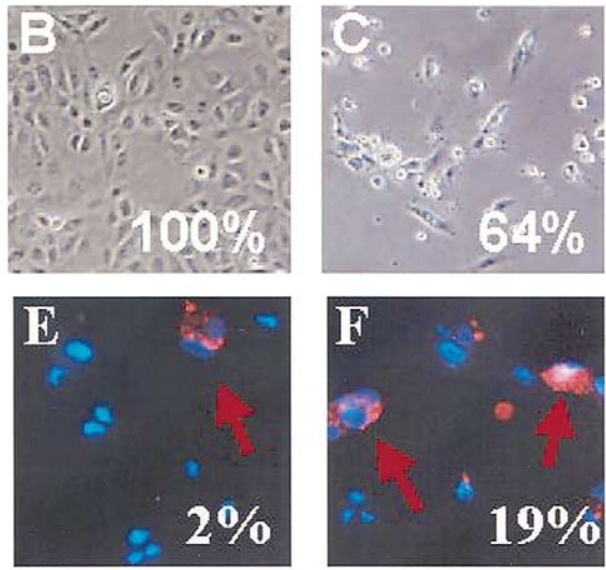

Figure 5. Ad-FADD infection induces apoptotic cell death in $\mathrm{NCl}-\mathrm{H} 226$ cells. $\mathrm{NCl}-\mathrm{H} 226$ cells plated were exposed to no virus (A), Ad-EGFP (B), or Ad-FADD (C) at MOI 50. Twenty-four hours later, cells were photographed under phase-contrast microscopy at original magnification $\times 10$. Cell viability was determined at 24 hours by crystal violet staining for a representative of three experiments performed in duplicate. Caspase activity was assessed by counting the percentage of $\mathrm{NCI}-\mathrm{H} 226$ cells stained with fluorescently labelled z-VAD (red, arrows) and Hoescht for nuclei (blue) 24 hours after infection with media (D), Ad-EGFP (E), or Ad-FADD (F) at MOI 25.

\section{Death Receptors<smiles>C1CCC1</smiles>

FADD<smiles>[AlH2]</smiles>

Caspase-8

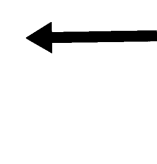

$\gamma$-irradiation Chemotherapy<smiles>[AlH2]</smiles>

p53

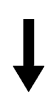

\section{Mitochondria} Caspase-9
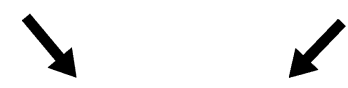

\section{Effector Caspases}<smiles>C1CCCC1</smiles>

\section{Apoptosis}

Figure 6. Role of FADD in mechanisms of apoptosis in tumor cells. Ionizing radiation and cytotoxic drugs induce apoptosis in cancer cells via a p53-dependent, mitochondrial pathway that activates caspase-9. In certain cell types, these cytotoxic agents can induce apoptosis via death-receptor and FADD-dependent signaling that activates caspase-8. Tumors can develop resistance to radiation and chemotherapy by altering the mitochondrial pathway (eg, p53 mutations as in $\mathrm{NCl}-\mathrm{H} 226$ cells) or changing death receptor expression and function (as in A549 cells). Overexpression of FADD may be used to activate latent apoptosis pathways in resistant clones or in combination with other therapeutic modalities.

We thank Brian Zuckerbraun, Deb Williams, and Andrea Gambotto for their help with this investigation.

\section{References}

1. Greenlee RT, Hill-Harmon MB, Murray T, Thun M. Cancer statistics, 2001. CA Cancer J Clin. 2001;51:15-36.

2. Allen RT, Hunter WJ III, Agrawal DK. Morphological and biochemical characterization and analysis of apoptosis. J Pharmacol Toxicol Methods. 1997;37:215-28.

3. Thompson CB. Apoptosis in the pathogenesis and treatment of disease. Science. 1995;267:1456-62.

4. Johnstone RW, Ruefli AA, Lowe SW. Apoptosis: a link between cancer genetics and chemotherapy. Cell. 2002;108:153-64.

5. Chinnaiyan AM, O'Rourke K, Tewari M, Dixit VM. FADD, a novel death domain-containing protein, interacts with the death domain of Fas and initiates apoptosis. Cell. 1995;81:505-12.

6. Boldin MP, Varfolomeev EE, Pancer Z, Mett IL, Camonis JH, Wallach D. A novel protein that interacts with the death domain of Fas/APO1 contains a sequence motif related to the death domain. J Biol Chem. 1995;270:7795-8.

7. Nagata S. Apoptosis by death factor. Cell. 1997;88:355-65.

8. Ashkenazi A, Dixit VM. Death receptors: signaling and modulation. Science. 1998;281:1305-8.

9. Martin DA, Siegel RM, Zheng L, Lenardo MJ. Membrane oligomerization and cleavage activates the caspase- 8 (FLICE/MACH $\alpha 1$ ) death signal. J Biol Chem. 1998;273:4345-9.

10. Muzio M, Stockwell BR, Stennicke HR, Salvesen GS, Dixit VM. An induced proximity model for caspase-8 activation. J Biol Chem. 1998; 273:2926-30.

11. Thornberry NA, Lazebnik Y. Caspases: enemies within. Science. 1998;281:1312-6.

12. Takahashi Y, Geller DA, Gambotto A, Watkins SC, Fung JJ, Murase N. Adenovirus-mediated gene therapy to liver grafts: successful gene transfer by donor pretreatment. Surgery. 2000;128:34552 . 
13. Li J, Bombeck CA, Yang S, Kim YM, Billiar TR. Nitric oxide suppresses apoptosis via interrupting caspase activation and mitochondrial dysfunction in cultured hepatocytes. J Biol Chem. 1999;274: 17325-33.

14. Kaufmann SH, Earnshaw WC. Induction of apoptosis by cancer chemotherapy. Exp Cell Res. 2000;256:42-9.

15. Reed JC. Dysregulation of apoptosis in cancer. J Clin Oncol. 1999; 17:2941-53.

16. Guchelaar HJ, Vermes A, Vermes I, Haanen C. Apoptosis: molecular mechanisms and implications for cancer chemotherapy. Pharm World Sci. 1997;19:119-25.

17. Fulda S, Meyer E, Friesen C, Susin SA, Kroemer G, Debatin KM. Cell type specific involvement of death receptor and mitochondrial pathways in drug-induced apoptosis. Oncogene. 2001;20:1063-75.

18. Green DR. Apoptotic pathways: paper wraps stone blunts scissors. Cell. 2000;102:1-4.

19. Newton K, Strasser A. Ionizing radiation and chemotherapeutic drugs induce apoptosis in lymphocytes in the absence of Fas or FADD/ MORT1 signaling: implications for cancer therapy. J Exp Med. 2000; 191:195-200.

20. Nicholson DW. From bench to clinic with apoptosis-based therapeutic agents. Nature. 2000;407:810-6.

21. Peter ME. The TRAIL DISCussion: it is FADD and caspase-8! Cell Death Differ. 2000;7:759-60.

22. Nambu Y, Hughes SJ, Rehemtulla A, Hamstra D, Orringer MB, Beer DG. Lack of cell surface Fas/APO-1 expression in pulmonary adenocarcinomas. J Clin Invest. 1998;101:1102-10.

23. Park SY, Billiar TR, Seol DW. Hypoxia inhibition of apoptosis induced by tumor necrosis factor-related apoptosis-inducing ligand (TRAIL). Biochem Biophys Res Commun. 2002;291:150-3.

24. Walczak H, Miller RE, Ariail K, Gliniak B, Griffith TS, Kubin M, et al. Tumoricidal activity of tumor necrosis factor-related apoptosisinducing ligand in vivo. Nat Med. 1999;5:157-63.

25. Lee SH, Shin MS, Kim HS, Lee HK, Park WS, Kim SY, et al. Alterations of the DR5/TRAIL receptor 2 gene in non-small cell lung cancers. Cancer Res. 1999;59:5683-86.

26. McDonald ER III, Chui PC, Martelli PF, Dicker DT, El Deiry WS Death domain mutagenesis of KILLER/DR5 reveals residues critical for apoptotic signaling. J Biol Chem. 2001;276:14939-45.

27. Pitti RM, Marsters SA, Lawrence DA, Roy M, Kischkel FC, Dowd P, et al. Genomic amplification of a decoy receptor for Fas ligand in lung and colon cancer. Nature. 1998;396:699-703.

28. Roth JA, Grammer SF, Swisher SG, Komaki R, Nemunaitis J, Merritt $\mathrm{J}$, et al. Gene therapy approaches for the management of non-small cell lung cancer. Semin Oncol. 2001;28(Suppl 14):50-6.

29. Dubensky TW. (Re-)Engineering tumor cell-selective replicating adenoviruses: a step in the right direction toward systemic therapy for metastatic disease. Cancer Cell. 2002;1:307-9.

30. Kim PK, Wang Y, Gambotto A, Kim YM, Weller R, Zuckerbraun BS, et al. Hepatocyte Fas-associating death domain protein/mediator of receptor-induced toxicity (FADD/MORT1) levels increase in response to pro-apoptotic stimuli. J Biol Chem. 2002;277:38855-62.

31. Kim PK, Weller R, Hua Y, Billiar TR. Ultraviolet irradiation increases FADD protein in apoptotic human keratinocytes. Biochem Biophys Res Commun. 2003;302:290-5.

\section{Discussion}

Dr Frank W. Sellke (Boston, Mass). You showed pretty clearly that you see increased activation of the caspases. Did you look at something such as TUNEL assay to be sure that you are actually seeing apoptosis rather than some inflammatory effect of gene transfer or some other mechanism causing the cell death?

Dr Kim. There are a lot of assays for apoptosis, and actually in our laboratory the results of TUNEL assay have been pretty variable. Even though we can see apoptosis by structure and activation or cleavage of caspases, we actually find with the caspase stain that I showed that we have a better assessment that caspases were indeed activated. Nevertheless, in these cell lines I have not done any assessment of DNA fragmentation by DNA laddering, TUNEL assay, or PARP cleavage.

Dr G. Alexander Patterson (St Louis, Mo). You acknowledge some of the limitations of the study. There may be another limitation. Is it correct that you studied two cell lines?

Dr Kim. Yes.

Dr Patterson. Do you have any comment about that and why those particular cell lines were selected, or do you have any preliminary data from other cell lines? In addition, I didn't understand exactly what the adenoviral control was and why you selected that control versus some other control.

Dr Kim. I will answer the second question first. We have used several controls. One would be an adenovirus that has no inserted gene, and the control I showed was an adenovirus that overexpresses the green fluorescent protein (Ad-EGFP). That is for two reasons. When we look under a fluorescent microscope we see at that dose (MOI 50) the adenovirus that overexpresses green fluorescent protein. We can show that infectivity occurred in more than $90 \%$ of the cells. In terms of nonspecific cell death induced by overexpressing any nonspecific protein, Ad-EGFP was the control that we used. An empty adenovirus (Ad-control) is just a control for nonspecific adenoviral toxicity.

As for the first question, we have actually done some preliminary studies in other cell lines. We have looked at a couple of adenocarcinoma cell lines, and we don't see as much cell death as we do in the squamous carcinoma cell lines; however, when you look at the green fluorescent protein expression in adenocarcinoma cells, there is less infectivity of those cells as well. So we are not sure if the adenovirus is not getting into the cells to express the FADD protein as an explanation for why we don't see as much cell death. So there is some variability between cell lines in terms of the induction of cell death. I chose to present the NCI-H226 cell line particularly because it made a pretty good movie in terms of demonstrating apoptotic cell death. 